Rend. Lincei Mat. Appl. 22 (2011), 3-15

DOI $10.4171 /$ RLM/584

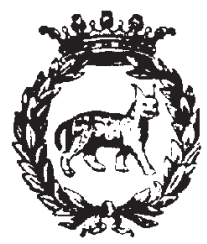

Partial Differential Equations - Osservazioni sui teoremi di inversione globale, by Antonio Ambrosetti.

Dedicato a Giovanni Prodi, grande maestro e caro amico.

\begin{abstract}
Some global inversion theorems with applications to semilinear ellitptic equation are discussed.
\end{abstract}

KEY WORDS: Local and global inversion, singularity theory, elliptic equations.

2000 Mathematics Subject Classification: 40E99, 32S, 35J25, 35J67

\title{
1. INTRODUZIONE
}

A partire dall'anno accademico 1970-1971 Giovanni Prodi tenne una serie di lezioni sull'Analisi Nonlineare, coprendo nell'arco di alcuni anni tutti i temi fondamentali di questa materia: dallo studio della geometria negli spazi di Banach, al grado topologico, alla biforcazione, ai metodi variazionali, fino ai sistemi dinamici e alla teoria ergodica.

Alla Scuola Normale Carlo Miranda aveva tenuto nel 1948-1949 un corso sul grado topologico [12], ma era la prima volta, in Italia, che venivano trattati in modo sistematico tutti questi argomenti. Prodi arricchiva le lezioni esponendo vari temi classici, fornendo dimostrazioni semplici e suggestive, e spesso discuteva applicazioni significative nuove o tratte da lavori di recente pubblicazione (a volte anche di preprint). Tutto questo, assieme alle straordinarie capacità didattiche di Prodi, sempre chiarissimo, ci affascinava e catturava l'entusiasmo di noi giovani ricercatori che vedevamo davanti a noi prospettive di grande suggestione.

Nel primo anno Prodi trattò la "geometria negli spazi di Banach" e, partendo dal calcolo differenziale in tali spazi, arrivò a discutere i problemi dell'inversione locale e globale di applicazioni in presenza di singolarità. Questi temi sono trattati nella prima parte di [13] e [3].

La teoria delle singolarità era stata sviuppata negli spazi euclidei, cfr. ad esempio $[16,17]$, ma non vi erano risultati per applicazioni negli spazi di Banach infinito dimensionali. Prodi affrontò dunque un tema completamente nuovo, dando un formidabile contributo alla teoria dell'inversione globale di funzioni tra spazi di Banach in presenza di singolarità. Come vedremo, questi risultati hanno permesso di studiare l'esistenza di soluzioni di una nuova classe di problemi al contorno ellittici con nonlinearità a-simmetriche. 
Per rendere omaggio a questo grande matematico italiano (che è stato il mio maestro e che poi ho avuto l'onore di avere come carissimo amico), vorrei esporre in questa breve Nota alcuni risultati su questi argomenti.

\section{Il Teorema di inversione globale di Hadamard-Caccioppoli}

Siano $X, Y$ due spazi di Banach e sia $F: X \rightarrow Y$ un'applicazione di classe $C^{1}$.

Un punto $u \in X$ è un punto singolare (per $F$ ) se il differenziale $F^{\prime}(u) \in$ $\mathscr{L}(X, Y)$ non è invertibile. L'insieme dei punti singolari di $F$ verrà indicato con $\Sigma_{F}$, o semplicemente con $\Sigma$. Se $u \notin \Sigma$, allora $u$ viene detto regolare (per $F$ ). Quello che segue è il classico teorema di inversione locale.

Teorema 2.1. Se $F \in C^{1}(X, Y)$ ed $u \notin \Sigma$ allora $F$ è localmente invertibile in $u$ : esistono due intorni $U$ di u e $V$ di $v=F(u)$ tra cui $F$ subordina un diffeomorfismo.

Per arrivare a dei risultati globali è opportuno assumere qualche condizione di compatezza. Ricordiamo che $F$ è propria se $F^{-1}(K)$ è compatto in $X$ per ogni compatto $K \subset Y$.

Lemma 2.2. Se $F \in C^{1}(X, Y)$ è propria e localmente invertibile in $X$, allora per ogni $h \in Y$ l'insieme delle soluzioni dell'equazione $F(u)=h, u \in X$, è finito e la sua cardinalità è localmente costante.

Come conseguenza si trova il classico teorema di inversione globale di Hadamard (in dimensione finita) e Caccioppoli.

Teorema 2.3 (di inversione globale). Se $F \in C^{1}(X, Y)$ è propria e $\Sigma=\emptyset$ allora $F$ è un diffeomorfismo globale di $X$ su $Y$. In particolare, l'equazione $F(u)=h$ ha una ed una sola soluzione in $X$ per ogni $h \in Y$.

Il teorema precedente può essere esteso al caso in cui $\Sigma \neq \emptyset$ sotto l'ipotesi che:

(i) $X \backslash F^{-1}(F(\Sigma))$ è connesso per archi;

(ii) $Y \backslash F(\Sigma)$ è semplicemente connesso.

TeOrema 2.4. Se $F \in C^{1}(X, Y)$ è propria e valgono le ipotesi (i-ii) precedenti, allora $F$ è un diffeomorfismo globale tra $X \backslash F^{-1}(F(\Sigma))$ e $Y \backslash F(\Sigma)$.

EsEmpio 2.1. Sia $\Omega$ un aperto limitato di $\mathbb{R}^{n}$ con frontiera regolare $\partial \Omega$. Poniamo $X=C_{0}^{2, v}(\Omega)$ (spazio delle funzioni di classe $C^{2, v}(\Omega) \cap C(\bar{\Omega})$ che si annullano su $\partial \Omega)$ e $Y=C^{0, v}(\Omega)$. e consideriamo l'applicazione $F: X \rightarrow Y$ definita da

$$
F(u)=-\Delta u-\alpha u+u^{3} .
$$

Allora le soluzioni di $F(u)=h, u \in X$ sono le soluzioni (classiche) del problema al contorno

$$
-\Delta u=\alpha u-u^{3}+h(x), \quad x \in \Omega, \quad u_{\partial \Omega}=0 .
$$


Poichè $F^{\prime}(u)$ è l'applicazione (lineare)

$$
v \rightarrow-\Delta v-\alpha v+3 u^{2} v, \quad v \in X
$$

$u \in \Sigma$ se e solo se l'equazione

$$
-\Delta v=\left(\alpha-3 u^{2}\right) v, \quad v \in X
$$

ha una soluzione non nulla, cioè se $\lambda_{j}\left[\alpha-3 u^{2}\right]=1$ per qualche $j \geq 2$. Qui e nel seguito $\lambda_{j}[a]$ indica il $j$-esimo autovalore di

$$
-\Delta v=\lambda a(x) v, \quad x \in \Omega, \quad v_{\partial \Omega}=0
$$

Se $a \equiv 1$ porremo $\lambda_{j}[a]=\lambda_{j}$.

Se $\alpha<\lambda_{1}$ allora $\alpha-3 u^{2}<\lambda_{1}$ e dalla proprietà di monotonia degli autovalori, segue subito che $\lambda_{j}\left[\alpha-3 u^{2}\right]>\lambda_{1}\left[\alpha-3 u^{2}\right]>1$ per ogni $j \geq 1$. Quindi, se $\alpha<\lambda_{1}$ si ha che $\Sigma=\emptyset$.

Sia ora $\alpha=\lambda_{1}$. Il ragionamento precedente implica che $\lambda_{1}\left[\alpha-3 u^{2}\right]=1$ se e solo se $u \equiv 0$. Dunque in questo caso $\Sigma=\{0\}$. Ovviamente $F(\Sigma)=\{0\}$ e quindi $Y \backslash F(\Sigma)=Y \backslash\{0\}$ è semplicemente connesso, cioè vale la (ii). Inoltre si può facilmente dimostrare che se $\alpha \leq \lambda_{1}$ l'equazione $F(u)=0$ ha solo la soluzione nulla: quindi $X \backslash F^{-1}(F(\Sigma))=X \backslash\{0\}$. Questo prova che anche la (i) è valida.

Infine si prova che $F$ è propria, mostrando che se $h_{k} \rightarrow h$ in $Y$, e se $u_{k} \in X$ è tale che $F\left(u_{k}\right)=h_{k}$, allora $u_{k}$ converge in $X$, a meno di sottosuccessioni. Questo dipende essenzialmente dal fatto che il problema (1) è coercivo e valgono le maggiorazioni a priori per le sue soluzioni.

Possiamo concludere, applicando i Teoremi 2.3 e 2.4 , che per $\alpha \leq \lambda_{1}$, la (1) ha una ed una sola soluzione per ogni $h \in Y$. Si verifica facilmente che lo stesso risultato si ottiene se si sostituisce $u^{3}$ con una $f \in C^{2}$ tale che $f(0)=f^{\prime}(0)=0$, $u f^{\prime \prime}(u)>0$ per $u \neq 0$ e

$$
\lim _{u \rightarrow+\infty} f(u)=\infty, \quad \lim _{u \rightarrow-\infty} f(u)=-\infty
$$

ESEMPIO 2.2. Consideriamo il problema

$$
-\Delta u=g(u)+h(x), \quad x \in \Omega, \quad u_{\partial \Omega}=0,
$$

e supponiamo che $g \in C^{1}(\mathbb{R})$ sia tale che $\lambda_{k}<g^{\prime}(u)<\lambda_{k+1}$ per qualche $k \geq 1$. In questo caso, $F(u)=-\Delta u-g(u)$ con $X$ ed $Y$ come nell'esempio precedente. Si ha:

$$
F^{\prime}(u)[v]=-\Delta v-g^{\prime}(u) v, \quad v \in X
$$


e $u \in \Sigma$ se e solo se $\lambda_{j}\left[g^{\prime}(u)\right]=1$ per qualche intero $j \geq 1$. Usiamo ancora la proprietà di monotonia degli autovalori: da $\lambda_{k}<g^{\prime}(u)$ segue che $\lambda_{k}\left[g^{\prime}(u)\right]<1$ mentre da $g^{\prime}(u)<\lambda_{k+1}$ segue che $\lambda_{k+1}\left[g^{\prime}(u)\right]>1$. Quindi anche in questo caso $\Sigma=\emptyset$. Se poi supponiamo che i limiti di $g^{\prime}(u)$ per $u \rightarrow \pm \infty$ non siano degli autovalori $\lambda_{j}$, allora $F$ è propria e quindi (2) ha una ed una sola soluzione per ogni $h \in Y$.

\section{TEOREMI DI INVERSIONE GLOBALE IN PRESENZA DI SINGOLARITÀ}

Nella seconda parte del suo corso alla Scuola Normale, Giovanni Prodi affrontò il caso in cui $\Sigma_{F}$ non è vuoto e sconnette $X$, discutendo un problema al contorno per una classe di equazioni differenziali ordinarie. Si trattava di un argomento del tutto originale che ha avuto come naturale sbocco un teorema generale di invertibilità globale in presenza di singolarità con applicazioni a problemi ellittici con nonlinearità a-simmetriche, studiato in [4].

L'idea è basata su questi passi:

1. studiare le proprietà geometriche di $\Sigma$ e di $F(\Sigma)$;

2. fare uno studio locale dell'equazione $F(u)=h$ in un intorno dei punti singolari;

3. usare il Lemma 2.2. per caratterizzare $F(X)$.

Supponiamo che $F \in C^{2}(X, Y)$ e che $\Sigma \neq \emptyset$. Diremo che $u \in \Sigma$ è un punto singolare di tipo (I) se

$$
\begin{gathered}
\exists \phi \in X: \operatorname{Ker}\left[F^{\prime}(u)\right]=\mathbb{R} \phi ; \\
\mathscr{R}:=F^{\prime}\left(u^{*}\right)[X] \text { e' chiuso ed esiste } z \in Y: Y=\mathbb{R} z \oplus \mathscr{R} .
\end{gathered}
$$

Indicata con $P$ la proiezione su $\mathbb{R} z$, esiste $\psi \in Y^{*}$ tale che

$$
P h=\langle\psi, h\rangle z .
$$

Se $u \in \Sigma$ è di tipo (I) allora si può trovare $\Gamma \in C^{1}(X, \mathbb{R})$ tale che in un intorno $B_{\varepsilon}$ di $u$ si ha

$$
\tilde{u} \in \Sigma \cap B_{\varepsilon} \quad \Leftrightarrow \quad \Gamma(\tilde{u})=0 .
$$

Inoltre

$$
\Gamma^{\prime}(u)[w]=\left\langle\psi, F^{\prime \prime}(u)[w, \phi]\right\rangle .
$$

Allora da (3) segue subito

Lemma 3.1. Sia $u \in \Sigma$ un punto singolare di tipo (I) e supponiamo che

$$
\exists w \in X:\left\langle\psi, F^{\prime \prime}(u)[w, \phi]\right\rangle \neq 0 .
$$


Allora $\Sigma \cap B_{\varepsilon}$ è una varietà di classe $C^{1}$, di codimensione 1 in $X$. Di conseguenza, se vale $\left(3^{\prime}\right)$ per ogni $u \in \Sigma$, allora $\Sigma$ è una varietà di classe $C^{1}$, di codimensione 1 in $X$.

Usando la definizione introdotta da Prodi, diremo che $u \in \Sigma$ è un punto singolare ordinario se è di tipo (I) e se

$$
\left\langle\psi, F^{\prime \prime}(u)[\phi, \phi]\right\rangle \neq 0
$$

Se $u \in X$ poniamo

$$
\Psi(u)=F(u)+\Gamma(u) z
$$

Se $\tilde{u} \in \Sigma$ è un punto singolare ordinario allora $\Psi^{\prime}(\tilde{u})$ è localmente invertible e $\Psi(\tilde{u})=F(\tilde{u})$. Posto, localmente in un intorno di $F(\tilde{u}), \gamma=\Gamma \circ \Psi^{-1}$ si ha che $g \in C^{1}, \gamma^{\prime} \neq 0$ e $v \in F(\Sigma) \Leftrightarrow \gamma(v)=0$. Quindi

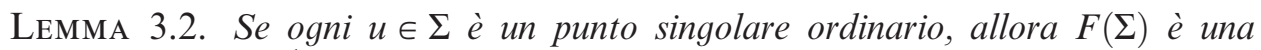
varietà di classe $C^{1}$, di codimensione 1 in $Y$.

Passiamo ora al punto 2 .

Lemma 3.3. Sia $u_{0} \in \Sigma$ un punto singolare ordinario e sia $\left\langle\psi, F^{\prime \prime}\left(u_{0}\right)[\phi, \phi]\right\rangle>0$, risp. $\left\langle\psi, F^{\prime \prime}\left(u_{0}\right)[\phi, \phi]\right\rangle\langle 0$. Allora esiste $\varepsilon>0$ tale che l'equazione $F(u)=v+s z$ con $P v=0$ ed $s \in \mathbb{R}, h a$ :

(a) 2 soluzioni se $0<s<\varepsilon$, risp. $-\varepsilon<s<0$;

(b) 1 soluzione se $s=0$;

(c) nessuna soluzione se $-\varepsilon<s<0$, risp. $0<s<\varepsilon$.

Possiamo ora applicare il Lemma 2.2 per trovare

Teorema 3.4. Supponiamo che $F \in C^{2}(X, Y)$ sia propria, che $\Sigma \neq \emptyset$ sia connesso, che consista di punti singolari ordinari e che l'equazione $F(u)=v$ abbia un'unica soluzione $\forall v \in F(\Sigma)$. Allora $\Sigma$, risp. $F(\Sigma)$, sono delle varietà $C^{1}$, connesse e di codimensione 1 in $X$, risp. $Y$ ed esistono due aperti connessi $Y_{0}, Y_{2} \subset Y$ tali che $F(u)=h$ ha:

(i) esattamente 2 soluzioni se $h \in Y_{2}$;

(ii) un'unica soluzione se $h \in F(\Sigma)$;

(iii) nessuna soluzione se $h \in Y_{0}$.

Con un'espressione suggestiva si può dire che $F$ piega $X$ lungo $\Sigma$. Per questo motivo, i punti singolari ordinari vengono detti pieghe (in inglese, "fold"). Si veda anche l'Osservazione 4.5. 

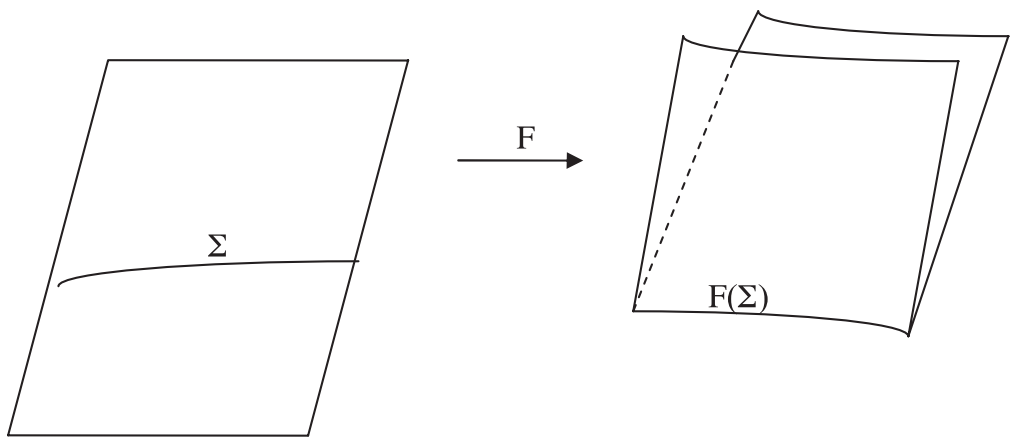

Piega

Esempio 3.1. Consideriamo nuovamente il problema (2) con $g \in C^{2}(\mathbb{R})$. Posto

$$
g_{-}=\lim _{u \rightarrow-\infty} \frac{g(u)}{u}, \quad g_{+}=\lim _{u \rightarrow+\infty} \frac{g(u)}{u}
$$

si abbia

$$
\begin{gathered}
-\infty<g_{-}<\lambda_{1}<g_{+}<\lambda_{2} ; \\
g^{\prime \prime}(u)>0, \quad \forall u \in \mathbb{R} .
\end{gathered}
$$

Usiamo le stesse notazioni dell'Esempio 2.2 ponendo $F(u)=-\Delta u-g(u), u \in X$. Un punto $u \in X$ è singolare se $F^{\prime}(u)[v]=-\Delta v-g^{\prime}(u) v$ ha una soluzione non identicamente nulla, cioè se $\lambda_{j}\left[g^{\prime}(u)\right]=1$ per qualche $j=1,2 \ldots$ Dalle ipotesi fatte su $g$ segue facilmente che $j=1$. Allora vale (Ii) $\operatorname{con} \phi=\phi_{1}$ dove $\phi_{1}$ è un'autofunzione relativa a $\lambda_{1}\left[g^{\prime}(u)\right]$ ( $\phi_{1}$ non cambia segno in $\Omega$ e quindi possiamo prendere $\left.\phi_{1}>0\right)$. Ovviamente vale anche (Iii) con $z=\phi_{1}$ e $\langle\psi, h\rangle=\int_{\Omega} h \phi_{1}$. In-
oltre, da $g^{\prime \prime}(u)>0$ segue anche che

$$
\left\langle\psi, F^{\prime \prime}(u)\left[\phi_{1}, \phi_{1}\right]\right\rangle=-\int_{\Omega} g^{\prime \prime}(u) \phi_{1}^{3}<0 .
$$

Allora ogni punto $u \in \Sigma$ è un punto singolare ordinario. Infine $F$ è propria (perchè $g_{ \pm}$non sono autovalori) e si mostra che l'equazione $F(u)=v$ ha un'unica soluzione per ogni $v \in F(\Sigma)$.

Indichiamo con (2) $)_{t}$ il problema (2) con $h=t \phi_{1}+w, \int_{\Omega} w \phi_{1}=0$. Usando il Teorema 3.4 si deduce che $\exists T \in \mathbb{R}$ tale che il problema $(2)_{t}$ ha esattamente 2 soluzioni, un'unica soluzione o nessuna soluzione a seconda che $t<T, t=T$, $t>T$.

OsSERVAZIONe 3.1. Molti autori hanno studiato il problema (2) $\operatorname{con} g$ tale che $g_{-}<\lambda_{1}<g_{+}$. I metodi usati sono di vario genere. Ad esempio, in [1] si usa il 
metodo delle sopra e sotto-soluzioni insieme col grado topologico, in [11] metodi variazionali, in [5] la teoria della biforcazione. In generale, non si suppone che $g$ sia convessa, ma si ottengono risultati del tipo: se $g_{-}<\lambda_{1}<g_{+}<+\infty, \exists T^{*} \in \mathbb{R}$ tale che $(2)_{t}$ non ha soluzioni per $t>T^{*}$ ed ha almeno 2 soluzioni, risp. almeno una soluzione, per $t<T^{*}$, risp. $t=T^{*}$. Usando la teoria dei punti critici si può assumere che $g_{+}=+\infty$ (fermo restando che $g_{-}<\lambda_{1}$ ). Precisamente

$$
\lim _{u \rightarrow+\infty} \frac{g(u)}{u^{p}}=c>0, \quad 1<p<\frac{n+2}{n-2}
$$

Il caso critico in cui $g(u) \sim u^{(n+2) /(n-2)}$ per $\rightarrow+\infty$ è stato studiato in [10]. Un'ampia bibliogarfia sull'argomento si trova in [9]. Ricordiamo anche l'estensione al p-laplaciano in [6].

\section{Risultati Di INVERSIONE GLOBALE IN PRESENZA Di CUSPIDi}

A differenza dello studio dei problemi ellittici con nonlinearità a-simmetrica, sono molto pochi i risultati relativi l'estensione del teorema astratto 3.4, cfr. ad esempio, [7, 14]; cfr. anche il survey [8] che contiene una ampia bibliografia. In particolare, in [14] viene studiata l'equazione $-\Delta u=\alpha u-u^{3}+h(x)$ (più precisamente, in [14] vengono assunte al bordo $\partial \Omega$ condizioni di Neumann omogenee, ma i rsultati valgono anche per condizioni di Dirichlet). La difficoltà principale sta nel fatto che $\Sigma_{F}$ contiene punti che sono del tipo (I) ma che possono non verificare la (4) e questo rende complicato lo studio di $\Sigma_{F}$ e soprattutto di $F\left(\Sigma_{F}\right)$.

Questi problemi non si presentano se consideriamo il problema omogeneo, cioe' $\operatorname{con} h=0$ :

$$
-\Delta u=\alpha u-f(u), \quad x \in \Omega, \quad u_{\partial \Omega}=0,
$$

con $\left(\right.$ cfr. Esempio 2.1) $f \in C^{2}(\mathbb{R})$ tale che $f(0)=f^{\prime}(0)=0, f^{\prime \prime}(u) u>0(u \neq 0)$ e

$$
\lim _{u \rightarrow+\infty} f(u)=\infty, \quad \lim _{u \rightarrow-\infty} f(u)=-\infty .
$$

OSSERVAZIONE 4.1. Se $f(u)=u^{3}$, si ottiene il problema (1). Si noti anche che come si è visto nell'Esempio 2.1, per $\alpha \leq \lambda_{1}$, (5) ha solo la soluzione banale $u=0$.

E' facile vedere che se $\alpha>\lambda_{1}$ l'equazione (5) ha almeno una soluzione positiva ed una negativa. Vogliamo ora determinare, nello spirito dei teoremi di inversione, il numero esatto delle soluzioni di (5).

Conviene considerare lo spazio di Hilbert $\mathscr{H}=L^{2}(\Omega)$ con prodotto scalare $(., .)_{\mathscr{H}}$. Indicato con $A$ l'inverso di $-\Delta$, con condizioni di Dirichlet su $\partial \Omega$, poniamo

$$
S_{\alpha}(u)=u-\alpha A u+A f(u), \quad u \in \mathscr{H} .
$$


in modo che le soluzioni di (5) si trovano risolvendo l'equazione $S_{\alpha}(u)=0$. Osserviamo che $S_{\alpha}^{\prime}(u)[v]=v-\alpha A v+A f^{\prime}(u) v$ e che $S_{\alpha}^{\prime \prime}(u)[v]=A f^{\prime \prime}(u) v$. Se $\lambda_{1}<$ $\alpha<\lambda_{2}$ si ha che $\Sigma_{S_{\alpha}} \neq \emptyset$. Inoltre, essendo ora $\langle\psi, h\rangle=\left(h, \phi_{1}\right)_{\mathscr{H}}$, si trova

$$
\left(S_{\alpha}^{\prime \prime}(u)\left[w, \phi_{1}\right], \phi_{1}\right)_{\mathscr{H}}=6 \int_{\Omega} f^{\prime \prime}(u) w \phi_{1}^{2} .
$$

Poichè $f^{\prime \prime}(u) u>0$ allora la condizione $\left(3^{\prime}\right)$ è verificata prendendo $w=u$. Dal Lemma 3.1 segue che $\Sigma_{S_{\alpha}}$ è una varietà connessa, regolare $\left(C^{\infty}\right)$, di codimensione 1 in $\mathscr{H}$ e quindi $\mathscr{H} \backslash \Sigma_{S_{\alpha}}$ ha due componenti connesse $C_{ \pm}$(per fissare le idee, indichiamo con $C_{-}$la componente che contiene 0$)$. Poichè $S_{\alpha}$ è una perturbazione compatta dell'identità, possiamo usare la teoria del grado topologico di LeraySchauder. Inoltre le soluzioni $u_{\alpha} \neq 0$ di $S_{\alpha}=0, \lambda_{1}<\alpha<\lambda_{2}$, sono non singolari, stanno in $C_{+}$e il loro indice di Leray-Schauder è dato da

$$
\operatorname{ind}\left(S_{\alpha}, u_{\alpha}\right)=1 \text {. }
$$

Sempre per $\lambda_{1}<\alpha<\lambda_{2}$ anche la soluzione $u \equiv 0$ è non singolare, sta in $C_{-} \mathrm{e}$

$$
\operatorname{ind}\left(S_{\alpha}, 0\right)=-1 \text {. }
$$

Inoltre il grado topologico $\operatorname{deg}\left(S_{\alpha}, B_{r}, 0\right)=1$, essendo $B_{r}$ il disco in $\mathscr{H}$, di centro l'origine e raggio $r$. Poniamo $\mathscr{S}_{\alpha}=S_{\alpha}^{-1}(0) \backslash\{0\}$. Osserviamo che $S_{\alpha}$ è propria e quindi $k:=\operatorname{card}\left[\mathscr{S}_{\alpha}\right]<\infty$. Dalle proprietà di additività del grado segue che per $\lambda_{1}<\alpha<\lambda_{2}$

$$
1=\operatorname{deg}\left(S_{\alpha}, B_{r}, 0\right)=\sum_{u_{\alpha} \in \mathscr{S}_{\alpha}} \operatorname{ind}\left(S_{\alpha}, u_{\alpha}\right)+\operatorname{ind}\left(S_{\alpha}, 0\right)=k-1
$$

Quindi $k=2$ e (5) ha esattamente 3 soluzioni: $u=0$ e 2 soluzioni, una positiva e una negativa. Si può anche estendere questo risultato ad $\alpha=\lambda_{2}$. Inoltre, se anche $\lambda_{2}$ è un autovalore semplice, le due soluzioni non banali trovate per $\alpha=\lambda_{2}$ possono essere continuate in un intorno destro di $\lambda_{2}$; inoltre da $\lambda_{2}$ si biforcano due rami di soluzioni che non possono coincidere con quelle precedenti.

In conclusione, tenuto anche conto dell'Osservazione 4.1, si può concludere col seguente teorema, che è un caso particolare di risultati più generali trovati in $[2]:$

TEOREMA 4.1. (i) Se $\alpha \leq \lambda_{1}$, (5) ha solo la soluzione banale $u=0$;

(ii) Se $\lambda_{1}<\alpha \leq \lambda_{2}$, (5) ha esattamente 2 soluzioni non banali, una positiva e una negativa;

(iii) Se $\lambda_{2}$ è semplice, $\exists \varepsilon>0$ tale che per $\lambda_{2}<\alpha<\lambda_{2}+\varepsilon$ il problema (5) ha, oltre alla soluzione banale $u=0$, esattamente 4 soluzioni non banali.

OSSERVAZIONE 4.2. Il teorema precedente può essere interpretato dal punto di vista della teoria della biforcazione. Dall'autovalore semplice $\lambda_{1}$ escono due rami connessi, illimitati $\mathscr{R}_{1}^{+}$e $\mathscr{R}_{1}^{-}$di soluzioni positive, risp. negative, di (5), tali che le proiezioni di $\mathscr{R}_{1}^{ \pm}$coprono la semiretta $\left(\lambda_{1},+\infty\right)$. Inoltre, per $\alpha \rightarrow \lambda_{2} \mathrm{i}$ due rami 
$\mathscr{R}_{1}^{ \pm}$si mantengono discosti da zero e non possono coincidere con i due rami $\mathscr{R}_{2}^{\prime}$, $\mathscr{R}_{2}^{\prime \prime}$ di biforcazione uscenti da $\lambda_{2}$ (supposto semplice).

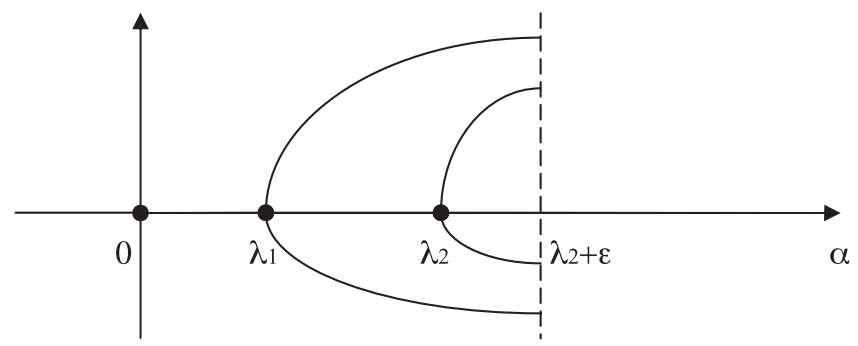

Diagramma di biforcazione di (5)

OsSERVAZIONE 4.3. Il Teorema 4.1 può essere esteso al problema non omogeneo

$$
\Delta u=\alpha u-f(u)+h, \quad u_{\partial \Omega}=0,
$$

con $\|h\| \ll 1$. Se $\alpha<\lambda_{2}+\varepsilon$ si prova che $\exists \delta=\delta(\alpha)>0$ tale che, se $\|h\|<\delta,\left(5^{\prime}\right)$ ha (esattamente) 1 soluzione per $\alpha \leq \lambda_{1}, 3$ soluzioni per $\lambda_{1}<\alpha \leq \lambda_{2}$ e 5 soluzioni per $\lambda_{2}<\alpha<\lambda_{2}+\varepsilon$.

Seguendo ora l'esposizione di [14], torniamo al problema (1). Come abbiamo visto, se $\alpha \in\left(\lambda_{1}, \lambda_{2}\right)$ allora $\Sigma_{S_{\alpha}} \neq \emptyset$, i punti singolari siano di tipo (I) e verificano l'equazione $\left(3^{\prime}\right)$.

OSSERVAZIONE 4.4. Se $\Omega \subset \mathbb{R}^{n}$ con $n \geq 2$, vi possono essere dei punti singolari che non sono punti singolari ordinari. Precisamente, ciò accade se e solo se

$$
\left(S_{\alpha}^{\prime \prime}(u)\left[\phi_{1}, \phi_{1}\right], \phi_{1}\right)_{\mathscr{H}}=0 .
$$

Infatti,

$$
\left(S_{\alpha}^{\prime \prime}(u)\left[\phi_{1}, \phi_{1}\right], \phi_{1}\right)_{\mathscr{H}}=-6 \int_{\Omega} u \phi_{1}^{3} .
$$

Inoltre può accadere, a differenza del caso in cui i punti di $\Sigma$ sono punti singolari ordinari, che vi siano $\bar{u} \in \mathscr{H},\|\bar{u}\|=1$ tali che il raggio $t \bar{u}, t>0$, non interseca $\Sigma$.

Indichiamo con C l'insieme dei punti $u \in \Sigma_{S_{\alpha}}$ che sono di tipo (I), verificano (3') e l'equazione (6). Ricordando che (cfr. sezione precedente) $u \in \Sigma_{S_{\alpha}} \Leftrightarrow \Gamma(u)=0$, ne segue che $\mathfrak{C}$ è individuato dalle equazioni

$$
\left\{\begin{array}{l}
\Gamma(u)=0 \\
\left(S_{\alpha}^{\prime \prime}(u)\left[\phi_{1}, \phi_{1}\right], \phi_{1}\right)_{\mathscr{H}}=0 .
\end{array}\right.
$$


Si può dimostrare (cfr. Prop. 2.5 di [14]) che, sotto certe condizioni, c è una sottovarietà di $\Sigma_{S_{\alpha}}$. Precisamente, indicatata con $z(w)$ la soluzione dell'equazione

$$
S_{\alpha}^{\prime}(u)[z]=-S_{\alpha}^{\prime \prime}(u)\left[w, \phi_{1}\right]
$$

si ha:

Lemma 4.2. Se esiste $w \in \mathscr{H}$ tale che valga $\left(3^{\prime}\right) e$

$$
\left(S_{\alpha}^{\prime \prime \prime}(u)\left[w, \phi_{1}, \phi_{1}\right], \phi_{1}\right)_{\mathscr{H}}+3\left(S_{\alpha}^{\prime \prime}(u)\left[\phi_{1}, z(w)\right], \phi_{1}\right)_{\mathscr{H}} \neq 0
$$

allora esiste $\varepsilon>0$ tale che $\mathfrak{C} \cap B_{\varepsilon}$ è una superficie regolare di codimensione 2 in $\mathscr{H}$.

Se $u \in \mathbb{C}$ è di tipo (I), verifica $\left(3^{\prime}\right),(6)$ e

$$
\left(S_{\alpha}^{\prime \prime \prime}(u)\left[\phi_{1}, \phi_{1}, \phi_{1}\right], \phi_{1}\right)_{\mathscr{H}}+3\left(S_{\alpha}^{\prime \prime}(u)\left[\phi_{1}, z\left(\phi_{1}\right)\right], \phi_{1}\right)_{\mathscr{H}} \neq 0,
$$

diremo che $u$ è una cuspide.

OSSERVAZIOne 4.5. Si può dimostrare che se $u^{*} \in \Sigma$ è una piega, esistono:

- uno spazio di Banach $Z \subset \mathscr{H}$,

- due intorni $U$ di $u^{*}$ e $V$ di $v^{*}=F\left(u^{*}\right)$,

- due diffeomorfismi $\sigma: U \rightarrow \mathbb{R} \times Z$ e $\tau: V \rightarrow \mathbb{R} \times Z$,

tali che, posto $\tilde{S}_{\alpha}=\tau \circ S_{\alpha} \circ \sigma^{-1}$, risulti

$$
\tilde{S}_{\alpha}(x, z)=\left(x^{2}, z\right)
$$

di modo che il seguente diagramma è commutativo

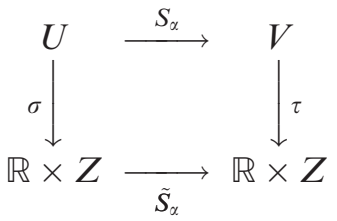

Analogamente, se $u^{*}$ è una cuspide, $S_{\alpha}$ è localmente equivalente a $\hat{S}_{\alpha}: \mathbb{R} \times \mathbb{R} \times Z$ $\rightarrow \mathbb{R} \times \mathbb{R} \times Z$,

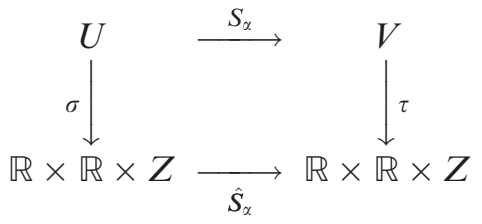


$\operatorname{con} \hat{S}_{\alpha}$ data da

$$
\hat{S}_{\alpha}(x, y, z)=\left(x^{3}-x y, y, z\right) .
$$

Le equazioni (9) e (10) giustificano i termini "piega" e "cuspide" introdotti prima.

Torniamo ora al problema (1).

TEOREMA 4.3. Esiste $\Lambda \in\left(\lambda_{1}, \lambda_{2}\right)$ tale che per $\lambda_{1}<\alpha<\Lambda, \Sigma_{S_{\alpha}}$ contiene una varietà $\tilde{C} \neq \emptyset$ regolare, di codimensione 2 , tale che:

(i) i punti di C sono delle cuspidi;

(ii) $\Sigma_{S_{\alpha}} \backslash \mathbb{C}$ ha due componenti connesse i cui punti sono dei punti singolari ordinari, e quindi dei punti di piega.

Dal teorema precedente si può dedurre un risultato riguardo all'esistenza di soluzioni di (1). Per endersi conto di quanto ci si può aspettare, usiamo la (10): risolvere $S_{\alpha}(u)=h$ equivale a trovare $(x, y, z) \in \mathbb{R} \times \mathbb{R} \times Z$ tali che $\left(x^{3}-x y, y, z\right)=(a, b, \zeta)=\tau(h)$, cioè $y=b, z=\zeta$ e $x$ verifica $x^{3}=a+b x$, equazione che ha una, 2 o 3 soluzioni al variare di $a, b \in \mathbb{R}$. Precisamente vale il seguente teorema.

TEOREMA 4.4. Esiste $\Lambda^{\prime}$ tale che se $\lambda_{1}<\alpha<\Lambda^{\prime}$ allora $\mathscr{H} \backslash F\left(\Sigma_{F}\right)$ è composto da due componenti connesse $\mathscr{H}_{1}, \mathscr{H}_{3}$ tali che

(i) il problema (1) ha esattamente 3 soluzioni se $h \in \mathscr{H}_{3}$;

(ii) ha una sola soluzione se $h \in \mathscr{H}_{1} \cup F(\mathfrak{C})$.

Inoltre

(iii) (1) ha esattamente 2 soluzioni se $h \in F(\Sigma) \backslash F(\mathfrak{C})$;

OsSERVAZIOni 4.6. (i) Nel caso di condizioni di Neumann al bordo (come considerato in [14]) si ha che $\lambda_{1}=0$ e si dimostra che si può prendere $\Lambda=\lambda_{2} / 7$ nel teorema 4.3 e $\Lambda^{\prime}=\lambda_{2} / 12$ nel Teorema 4.4 .

(ii) In generale, il risultato del Teorema 4.4 non può essere esteso a tutto $\left(\lambda_{1}, \lambda_{2}\right)$. Infatti, nel caso in cui $\Omega$ è l'intervallo $(0,1)$ e per condizioni di Neumann al bordo, è dimostrato in [15] che esiste $\lambda^{*} \in\left(\lambda_{2} / 12, \lambda_{2}\right)$ tale che (1) ha, per $\alpha \in\left(\lambda^{*}, \lambda_{2}\right)$, almeno 5 soluzioni per certi termini forzanti $h \in C^{0, v}$.

Anche se sono stati ottenuti vari risultati di notevole interesse, alcuni dei quali discussi in questa Nota, molto resta ancora da fare sulla teoria della singolarità negli spazi di Banach e sulle applicazioni ai problemi ellittici semiilineari. Qui ci limitiamo ad indicare alcune questioni aperte:

(a) studiare il caso periodico per un'equazione ordinaria del secondo ordine con $g$ verificante (g.1) ed $h$ periodica (questo problema fu posto dallo stesso Prodi nel suo corso); 
(b) determinare delle condizioni su una generica $F: X \rightarrow Y$, regolare, non necessariamente derivante da (1), perchè valga il Teorema 4.3 e studiare l'invertibilità globale di $F$ in presenza di cuspidi;

(c) trovare una dimostrazione semplice dei risultati enunciati nei Teoremi 4.3 e 4.4, relativi all'equazione (1) con condizioni di Neumann al bordo, per $\alpha \in\left(\lambda_{1}, \lambda_{1}+\varepsilon\right)$, con $\varepsilon>0$ sufficientemente piccolo;

(d) provare che, se tutti gli autovalori $\lambda_{k}$ sono semplici, $\lambda_{k}<\lambda<\lambda_{k+1}$, l'equazione (3) ha esattamente $2 k$ soluzioni non banali o trovare dei contro-esempi.

\section{REFERENCES}

[1] H. Amann - P. Hess, Multiplicity results for a class of elliptic boundary value problems, Proc. Royal Soc. Edinburgh, 84 (1979), 145-151.

[2] A. Ambrosetti - G. Mancini, Sharp nonuniqueness results for some nonlinear problems, J. Nonlin. Anal. TMA, 3-5 (1979), 635-645.

[3] A. Ambrosetti - G. Prodi, A Primer of Nonlinear Analysis, Cambridge Studies in Advanced Mathematics No. 34, Cambridge Univ. Press, 1993.

[4] A. Ambrosetti - G. Prodi, On the inversion of some differentiable mappings with singularities between Banach spaces. Ann. Mat. Pura ed Appl. 93 (1972), 231246.

[5] D. Arcoya - J. Carmona, On the so-called Ambrosetti problem, J. Eur. Math. Soc. 8(2) (2006), 181-188.

[6] D. Arcoya - D. Ruiz, The Ambrosetti-Prodi problem for the p-laplace operator. Comm. P.D.E. 31 (2006), 849-865.

[7] M. Berger - P. T. Church - J. G. Timourian, Folds and cusps in Banach spaces with applications to nonlinear partial differential equations, Indiana Univ. Math. J. 34 (1985), 1-19.

[8] P. T. Church - J. G. Timourian, Global structure for nonlinear operators in differential and integral equations I. Folds, e Global structure for nonlinear operators in differential and integral equations II. Cusps, in Topological nonlinear analysis II: degree, singularities and variations, M. Matzeu - A. Vignoli Ed. PNDEA v.27, Birkhauser, 1996.

[9] D. G. De Figueiredo, Lectures on Boundary Value Problems of Ambrosetti-Prodi Type, 12th Brazilian Seminar of Analysis, São Paulo, Brasil, 1980.

[10] D. G. De Figueiredo - Yang Jianfu, Critical superlinear Ambrosetti-Prodi problems, Top. Meth. Nonlin. Anal.s Journal 14 (1999), 59-80.

[11] D. G. De Figueiredo - S. Solimini, A variational approach to superlinear elliptic problems, Comm. P.D.E. 9 (1984), 699-717.

[12] C. Miranda, Problemi di esistenza in analisi funzionale, Pubbl. Classe di Scienze, Scuola Normale Sup. Pisa, 1975 (ristampa).

[13] G. Prodi - A. Ambrosetti, Analisi non lineare. Quad. I, Pubbl. Classe di Scienze, Scuola Normale Sup. Pisa, 1973.

[14] B. Ruf, Singularity theorey and the geometry of a nonlinear elliptic equation, Ann. Sc. Norm. Sup. Pisa, 17 (1990), 1-33.

[15] B. RuF, Forced secondary bifurcation in an elliptic boundary value problem, Diff. Int. Eq. 5-4 (1992), 793-804.

[16] R. Тном, Les singularités des applications différentiables, Ann. Inst. Fourier 6 (1956), $43-87$. 
[17] H. Whitney, On singularities of mappings of Euclidean spaces I. Mappings of the plane into the plane, Ann. Math. 62 (1955), 374-410.

Received 1 September 2010,

and in revised form 24 September 2010.

S.I.S.S.A.

via Bonomea 265, 34136 Trieste

ambr@sissa.it 
